\title{
CONCEPT OF STATISTICAL CAUSALITY AND LOCAL MARTINGALES
}

\author{
Dragana Valjarević ${ }^{1 *}$, Ljiljana Petrović ${ }^{2}$ \\ ${ }^{1}$ Faculty of Mathematics and Sciences, University of Priština, Kosovska Mitrovica, Serbia. \\ ${ }^{2}$ Department of Mathematics and Statistics, Faculty of Economics, University of Belgrade, Belgrade, Serbia.
}

\begin{abstract}
In this paper we consider a statistical concept of causality in continuous time in filtered probability spaces which is based on Granger's definitions of causality. The given causality concept is closely connected to the preservation of the property being

a local martingale if the filtration is getting larger. Namely, the local martingale remains unpredictable if the amount of information is increased. We proved that the preservation of this property is equivalent with the concept of causality.
\end{abstract}

Key words: Causality, filtration, local martingale, martingale.

\section{INTRODUCTION}

One of the goals of science is to find causal relations. This cannot always be done by experiments and researchers are restricted to observing the system they want to describe. This is the case in economics, demography and many other fields. Causality concepts expressed in terms of orthogonality in Hilbert spaces of square integrable random variables was studied by (Hosoya, 1977), (Florens \& Mouchart, 1985). In the papers (Florens \& Mouchart, 1982), (Mykland, 1986), (Gill \& Petrović, 1987) and (Petrović, 1996) it is shown how conditional independence can serve as a basis for a general probabilistic theory of causality for both processes and single events.

Linear Granger--causality was introduced by (Granger, 1969). We shall study a nonlinear version of the concept. Like the linear one, it defines that the process $\mathbf{Y}=\left\{Y_{t}, t \in I\right\},(I \subseteq \mathbf{R})$ does not cause the process $\mathbf{X}=\left\{X_{t}, t \in I\right\}$ if, for all $t$, the orthogonal projection of the $\mathrm{L}^{2}$-space representing $X_{s}, s>t$ on the space representing $X_{s}$ and $Y_{s}, s \leq t$ is contained in the space representing $X_{s}, s \leq t$. However, the spaces representing stochastic variables are those over the $\sigma$ field generated by these variables, while in the linear case they are the smallest closed linear spaces containing the variables. The concept was first suggested in (Granger \& Newbold, 1977) and studied by (Chamberlain, 1982) and (Florens \& Mouchart, 1982).

The study of Granger--causality has been mainly preoccupied with time series. We shall instead concentrate on continuous time processes. Many of systems to which it is natural to apply tests of causality, take place in continuous time. For example, this is generally the case within economy. In this case, it may be difficult to use a discrete time model. Also, the observed "causality" in a discrete time model may depend on the length of interval between each two successive samplings, as in the case with Granger-causality as shown, for example, by (McCrorie \& Chambers, 2006).

This concept of causality is closely connected to the notion of extremality of measures and martingale problem (Petrović \& Stanojević, 2010). Also, the given causality concept is related to the stable subspaces of $H^{p}$ which contains right continuous modifications of martingales (Petrović \& Valjarević, 2013) and with orthogonality of martingales (Valjarević \& Petrović, 2012).

One of the aim of this paper is to give insight in known results which concern already mention concept of causality, but also to give some new. Results are mainly preoccupied with preservation of the local martingale property when the filtration is getting larger.

After Introduction, in the second part of the paper we give various concepts of causality relationship between flow of information (represented by filtrations). Also, we give a generalization of a causality relationship " $\mathrm{G}$ entirely causes $\mathrm{H}$ within $\mathrm{F}$ " which (in terms of $\sigma$-algebras) was first given in (Mykland, 1986) and which is based on Granger's 
definition of causality from (Granger, 1969) and discuss the relationship to nonlinear Granger-causality.

In the third part we give some preliminaries on martingales where we consider the connection between the concept of causality and preservation of martingale property. The similar statement holds for local martingales, too.

\section{PRELIMINARIES AND NOTATIONS}

Let $(\Omega, \mathcal{F}, P)$ be an arbitrary probability space and let $\mathrm{F}=\left\{\mathcal{F}_{t}, t \in I(\subseteq \mathrm{R})\right\}$, be a family of sub $\sigma$-algebras of $\mathcal{F}$. Then $\mathcal{F}_{t}$ can be interpreted as the set of events observed up to time $t .\left(\mathcal{F}_{\infty}\right)$ is the smallest $\sigma$-algebra containing all the $\mathcal{F}_{t}$ (even if sup $I<+\infty)$. So, we have $\mathcal{F}_{\infty}=\bigvee_{t \in I} \mathcal{F}_{t}$.

A filtration $\mathbf{F}=\left\{\mathcal{F}_{t}, t \in I\right\}$, is a nondecreasing family of $\sigma$-subalgebras of $\mathcal{F}$, that is:

$$
\mathcal{F}_{s} \subseteq \mathcal{F}_{t}, s \leq t
$$

A probabilistic model for a time-dependent system is described by $\left(\Omega, \mathcal{F}, \mathcal{F}_{t}, P\right)$ where $(\Omega, \mathcal{F}, P)$ is a probability space and $\left\{\mathcal{F}_{t}, t \in I\right\}$ is a "framework" filtration, i.e. $\mathcal{F}_{t}$ is a set of all events in the model up to and including time $t$ and $\mathcal{F}_{t}$ is a subset of $\mathcal{F}$. We suppose that the filtration $\left(\mathcal{F}_{t}\right)$ satisfies the "usual conditions", which means that $\left(\mathcal{F}_{t}\right)$ is right continuous and each $\left(\mathcal{F}_{t}\right)$ is complete.

Analogous notation will be used for filtrations $\mathrm{H}=\left\{\mathcal{H}_{t}\right\}, \mathrm{G}=\left\{\mathcal{G}_{t}\right\}$, and $\mathrm{J}=\left\{\mathcal{J}_{t}\right\}$

Possibly the weakest form of causality can be introduced in the following way.

Definition 2.1 It is said that $H$ is submitted to $G$ or that $\mathrm{H}$ is a subfiltration of $\mathrm{G}$ (and written as $\mathrm{H} \subseteq \mathrm{G}$ ) if $\mathcal{H}_{t} \subseteq \mathcal{G}_{t}$ for each $t$.

It will be said that the filtrations $H$ and $G$ are equivalent (and written as $\mathrm{H}=\mathrm{G}$ ) if $\mathrm{H} \subseteq \mathrm{G}$ and $\mathrm{G} \subseteq$ H.

A family of $\sigma$-algebras induced by a stochastic process $\mathrm{X}=\left\{X_{t}, t \in I\right\}$, is given by $\mathrm{F}^{\mathrm{X}}=\left\{\mathcal{F}_{t}^{X}, t \in I\right\}$, where:

$$
\mathcal{F}_{t}^{X}=\sigma\left\{X_{u}, u \in I, u \leq t\right\},
$$

being the smallest $\sigma$-algebra with respect to which the random variables $X_{u}, u \leq t$ are measurable. The process $X_{t}$ is $\left(\mathcal{F}_{t}\right)$-adapted if $\mathcal{F}_{t}^{X} \subseteq \mathcal{F}_{t}$ for each $t$.

A family of $\sigma$-algebras may be induced by several processes, e.g. $\mathrm{F}^{\mathrm{X}, \mathrm{Y}}=\left\{\mathcal{F}_{t}^{X, Y}, t \in I\right\}$ where:

$$
\mathcal{F}_{t}^{X, Y}=\mathcal{F}_{t}^{X} \bigvee \mathcal{F}_{t}^{Y}, t \in I .
$$

On the probability space $(\Omega, \mathcal{F}, P)$ the process $\mathrm{Z}=\left\{Z_{t}, t \in I\right\}$, is a $\left(\mathcal{F}_{t}, P\right)-$ martingale if $Z_{t}$ is $\left(\mathcal{F}_{t}\right)$-adapted and $Z_{s}=E\left(Z_{t} \mid \mathcal{F}_{s}\right)$ for all $t \geq s$.

Definition 2.2 (compare with (Rozanov, 1974)) Let $(\Omega, \mathcal{F}, P)$ be a probability space and $\mathcal{F}_{1}, \mathcal{F}_{2}$ and $\mathcal{G}$ arbitrary $\sigma-$ subalgebras from $\mathcal{F}$. It is said that $\mathcal{G}$ is splitting for $\mathcal{F}_{1}$ and $\mathcal{F}_{2}$ or that $\mathcal{F}_{1}$ and $\mathcal{F}_{2}$ are conditionally independent given $\mathcal{G}$ (and written as $\left.\mathcal{F}_{1} \perp \mathcal{F}_{2} \mid \mathcal{G}\right)$ if:

$$
\begin{gathered}
\left(\forall A_{1}\right)\left(A_{1} \in \mathcal{F}_{1}\right)\left(\forall A_{2}\right)\left(A_{2} \in \mathcal{F}_{2}\right) P\left(A_{1} A_{2} \mid \mathcal{G}\right) \\
=P\left(A_{1} \mid \mathcal{G}\right) P\left(A_{2} \mid \mathcal{G}\right) .
\end{gathered}
$$

The following results give an alternative way of defining splitting.

Lemma 2.1 (see (Gill \& Petrović, 1987)) $\mathcal{F}_{1} \perp \mathcal{F}_{2} \mid \mathcal{G}$ if and only if $\mathcal{F}_{1} \perp \mathcal{F}_{2} \mid \mathcal{G}$ if and only if $E\left(\mathcal{F}_{i} \mid \mathcal{F}_{j} \vee \mathcal{G}\right) \subseteq \mathcal{G}$ for $i, j=1,2, i \neq j$.

Corollary 2.2 (Petrović and Stanojević, 2005) $\mathcal{F}_{1} \perp \mathcal{F}_{2} \mid \mathcal{G}$ if and only if $\mathcal{F}^{\prime}{ }_{1} \perp \mathcal{F}^{\prime}{ }_{2} \mid \mathcal{G}$ for all $\mathcal{F}^{\prime}{ }_{i} \subseteq \mathcal{F}_{i} \vee \mathcal{G}, i=1,2$.

The intuitively plausible notion of causality formulated in terms of Hilbert spaces is given in (Petrović, 1989). We shall use its analogous in terms of filtrations.

Let J, G and $\mathrm{H}$ be arbitrary filtrations. We can say that " $\mathrm{G}$ is a cause of $\mathrm{J}$ within $\mathrm{H}$ " if:

$$
\mathcal{J}_{\infty} \perp \mathcal{H}_{t} \mid \mathcal{G}_{t}
$$

because the essence of (1) is that all information about $\left(\mathcal{J}_{\infty}\right)$ that gives $\left(\mathcal{H}_{t}\right)$ comes via $\left(\mathcal{G}_{t}\right)$ for arbitrary $t$; equivalently, $\left(\mathcal{G}_{t}\right)$ contains all information from the $\left(\mathcal{H}_{t}\right)$ needed for predicting $\left(\mathcal{J}_{\infty}\right)$. According to Corollary 2.2 , is equivalent to $\mathcal{J}_{\infty} \perp \mathcal{H}_{t} \vee \mathcal{G}_{t} \mid \mathcal{G}_{t}$ The last relation means that the condition $\mathrm{G} \subseteq \mathrm{H}$ does not represent essential restriction. Thus, it was natural to introduce the following definition of causality between filtrations.

Definition 2.3 (Petrović, 1989) It is said that G entirely causes (or just causes) $\mathrm{J}$ within $\mathrm{H}$ relative to $\mathrm{P}$ (and written as $\mathrm{J} \mid<\mathrm{G} ; \mathrm{H} ; \mathrm{P}$ ) if $\mathcal{J}_{\infty} \subseteq \mathcal{H}_{\infty}, \mathrm{G} \subseteq \mathrm{H}$ and if $\left(\mathcal{J}_{\infty}\right)$ is conditionally independent of $\left(\mathcal{H}_{t}\right)$ given $\left(\mathcal{G}_{t}\right)$ for each $t$, i.e.

$$
\mathcal{J}_{\infty} \perp \mathcal{H}_{t} \mid \mathcal{G}_{t}
$$

(i.e. $\mathcal{J}_{u} \perp \mathcal{H}_{t} \mid \mathcal{G}_{t}$ holds for each $t$ and each $u$ ), or:

$$
\left(\forall A \in \mathcal{J}_{\infty}\right) \quad P\left(A \mid \mathcal{H}_{t}\right)=P\left(A \mid \mathcal{G}_{t}\right) .
$$

If there is no doubt about $P$, we omit "relative to $P$ ". 
Intuitively, $\mathrm{J} \mid<\mathrm{G} ; \mathrm{H} ; \mathrm{P}$ means that, for arbitrary $t$, information about $\left(\mathcal{J}_{\infty}\right)$ provided by $\left(\mathcal{H}_{t}\right)$ is not "bigger" than that provided by $\left(\mathcal{G}_{t}\right)$.

A definition, similiar to Definition 2.3 was first given in (Mykland, 1986). However, the definition from (Mykland, 1986) contains also the condition $\mathrm{J} \subseteq \mathrm{H}$ (instead of $\mathcal{J}_{\infty} \subseteq \mathcal{H}_{\infty}$ ) which does not have intuitive justification. Since Definition 2.3 is more general then the definition given in (Mykland, 1986), all results related to causality in the sense of Definition 2.3 will be true and in the sense of the Hilbert space version of the definition from (Mykland, 1986), when we add the condition $\mathrm{J} \subseteq \mathrm{H}$ to them.

If $\mathrm{G}$ and $\mathrm{H}$ are such that $\mathrm{G} \mid<\mathrm{G}$; $\mathrm{G} \vee \mathrm{H}$; $\mathrm{P}$ (where $\mathrm{G} \vee \mathrm{H}$ is a family determined by $\left.(\mathcal{G} \vee \mathcal{H})_{t}=\mathcal{G}_{\mathrm{t}} \vee \mathcal{H}_{\mathrm{t}}\right)$, we shall say that $\mathrm{H}$ does not cause $\mathrm{G}$. It is clear that the interpretation of, Granger--causality is now that $\mathrm{H}$ does not cause G if $\mathrm{G} \mid<\mathrm{G}$; G V H; P (see (Mykland, 1986)). Without difficulty, it can be shown that this term and the term " $\mathrm{H}$ does not anticipate $\mathrm{G}$ " (as introduced in (Rozanov, 1977)) are identical.

If $G$ and $H$ are such that $G \mid<G$; $H$; $P$ we shall say that $\mathrm{G}$ is its own cause within $\mathrm{H}$ (compare with (Mykland, 1986)). It should be mentioned that the notion of subordination (as introduced in (Rozanov, 1974)) is equivalent to the notion of being one's own cause, as defined here.

These definitions can be applied to stochastic processes. It will be said that stochastic processes are in a certain relationship if and only if the corresponding induced filtrations are in this relationship. For example, $\left(\mathcal{F}_{t}\right)$-adapted stochastic process $X_{t}$ is its own cause if $\mathrm{F}^{X}=\left(\mathcal{F}_{t}^{X}\right)$ is its own cause within $\mathrm{F}=\left(\mathcal{F}_{t}\right)$ i.e. if:

$$
\mathrm{F}^{X} \text { < } \mathrm{F}^{X} ; \mathrm{F} ; P \text {. }
$$

We shall give some properties of causality relationship from Definition 2.3 which will be needed later.

Lemma 2.3 (see (Petrović, 1989)) From J K G; H; $P$ and $\mathrm{J} \subseteq \mathrm{H}$ it follows that $\mathrm{J} \subseteq \mathrm{G}$.

The following result shows that a process $X_{t}$ which is its own cause is completely described by its behavior relative to $\mathrm{F}^{X}$.

Lemma 2.4 (Petrović \& Stanojević, 2010) $X=$ $\left\{X_{t}, t \in I\right\}$, is a Markov process relative to filtration $\mathrm{F}=\left(\mathcal{F}_{t}, t \in I\right)$ on a filtered probability space $\left(\Omega, \mathcal{F}, \mathcal{F}_{t}, P\right)$ if and only if $X_{t}$ is a Markov process (relative to $\mathrm{F}^{X}$ and the process is its own cause within relative to $\$ P \$$.

Mathematics
Corollary 2.5 (Petrović \& Stanojević, 2010) Brownian motion $\mathrm{W}=\left\{W_{t}, t \in I\right\}$ on a filtered probability space $\left(\Omega, \mathcal{F}, \mathcal{F}_{t}, P\right)$ is its own cause within $\mathrm{F}=\left(\mathcal{F}_{t}, t \in I\right)$ relative to probability $P$.

From the following result it follows that relationship "being one's own cause" is the transitive relationship.

Lemma 2.6 (compare with (Petrović, 1989)) From G $\mathrm{K} \mathrm{G} ; \mathrm{H} ; P$ and $\mathrm{H} K \mathrm{H} ; \mathrm{J} ; P$ it follows that $\mathrm{G} \mathrm{K}$

G; J; $P$.

The following result gives the invariance under convergence for causality relationship from Definition 2.3 .

Proposition 2.7 (Petrović \& Dimitrijević, 2011) In probability space $(\Omega, \mathcal{F}, P)$ let $\mathrm{F}$ and $\mathrm{G}$ be a filtrations. Let $\left\{X^{(n)}\right\}$ is a sequence of stochastic processes satisfying.

$$
X_{t}^{(n)} \stackrel{P}{\rightarrow} X_{t} \text { when } n \rightarrow \infty
$$

for every $t \in I \subseteq R$ and

$$
\mathrm{F}^{X^{(n)}}<\mathrm{G} ; \mathrm{F} ; P \text {, for every } n
$$

Then for the process $X_{t}$ holds

$$
\mathrm{F}^{X} \text { < G; F; } P \text {. }
$$

Remark. For some results from part 2, the proofs in given literature are in terms of Hilbert spaces. The proofs are analogous in the $\sigma$-algebra concept.

Proposition 2.8 (Mykland, 1986) Let $\mathrm{G}=\left(\mathcal{G}_{t}\right), \mathrm{H}=$ $\left(\mathcal{H}_{t}\right), \mathrm{J}=\left(\mathcal{J}_{t}\right)$ and $\mathrm{F}=\left(\mathcal{F}_{t}\right)$ be filtrations in a probability space Then the following statements are equivalent:

(i) $\mathrm{J} K \quad \mathrm{G} ; \mathrm{H} ; P$ and $J \mathrm{~K} \quad \mathrm{H} ; \mathrm{F} ; P$;

(ii) $\mathrm{J} K \mathrm{G} ; \mathrm{F} ; P$ and $\mathrm{G} \subseteq \mathrm{H} \subseteq \mathrm{F}$.

Lemma 2.9 (Mykland, 1986) In the measurable space $(\Omega, \mathcal{F}$,$) let the filtrations \mathrm{H}=\left\{\mathcal{H}_{t}\right\}, \mathrm{G}=\left\{\mathcal{G}_{t}\right\}$, and $\mathrm{J}=\left\{\mathcal{J}_{t}\right\}$ be given and let $P$ and $Q$ be probability measures on $\mathcal{F}$ satisfying $\mathrm{Q} \ll P$ with $\frac{d Q}{d P}$ as $\left(\mathcal{H}_{\infty}\right)$-measurable. Then:

$$
\text { J K G;H;P } \quad \Rightarrow \text { J K G;H; Q. }
$$

Given causality concept links Granger--causality with the concept of adapted distribution, which have been introduced by Kiesler and Hoover in $\backslash$ cite $\{\mathrm{KH}\}$. 


\section{CAUSALITY AND MARTINGALE PROPERTY}

This section is concerned with the analysis of the connection between the preservation of the martingale property and the concept of causality.

The martingale property remains valid if the filtration decreases, but in general this property is not preserved if the filtration increases. When the filtration is getting larger, the preservation of the martingale property is strongly connected to the concept of causality, because martingale is the process which remains unpredictable even if the information $\sigma$-algebra increase.

Let $\left(\Omega, \mathcal{F}, \mathcal{F}_{t}, P\right), t \in I=[0,+\infty)$ be a filtered probability space with $\mathrm{F}=\left(\mathcal{F}_{t}, t \in I\right)$ right continuous and complete.

Let $H$ be a set of right continuous modifications of the $\left(\mathcal{H}_{t}\right)$-adapted processes of the form

$$
H=\left\{M_{t}: M_{t}=P\left(A \mid \mathcal{H}_{t}\right), A \in \mathcal{H}_{\infty}\right\} .
$$

Then, the following result holds.

Theorem 3.1 Suppose that $\mathcal{H}_{\infty} \subseteq \mathcal{F}_{\infty}$ and $\mathrm{G} \subseteq \mathrm{F}$. Then $\mathrm{H} K \mathrm{G} ; \mathrm{F} ; P$ if and only if every $\left(\mathcal{H}_{t}\right)$-adapted, $\left(\mathcal{G}_{t}\right)$-martingale is $\left(\mathcal{F}_{t}\right)$ - martingale.

Proof. Suppose that H $K$ G; F; $P$. Then $\mathcal{H}_{\infty} \subseteq$ $\mathcal{F}_{\infty}, \mathrm{G} \subseteq \mathrm{F}$ and

$$
\left(\forall A \in \mathcal{H}_{\infty}\right) \quad P\left(A \mid \mathcal{G}_{t}\right)=P\left(A \mid \mathcal{F}_{t}\right) .
$$

Suppose that elements of $H$, of the form (2) are $\mathcal{G}_{t}$-martingales so:

$$
\begin{aligned}
M_{t} & =E\left(M_{\infty} \mid \mathcal{G}_{t}\right)=E\left(P\left(A \mid \mathcal{H}_{\infty}\right) \mathcal{G}_{t}\right) \\
& =E\left(E\left(\chi_{A} \mid \mathcal{H}_{\infty}\right) \mathcal{G}_{t}\right)=\mathrm{E}\left(\chi_{A} \mid \mathcal{G}_{t}\right) \\
& =P\left(A \mid \mathcal{G}_{t}\right)=P\left(A \mid \mathcal{F}_{t}\right)=E\left(\chi_{A} \mid \mathcal{F}_{t}\right) \\
& =E\left(E\left(\chi_{A} \mid \mathcal{H}_{\infty}\right) \mid \mathcal{F}_{t}\right) \\
& =E\left(P\left(A \mid \mathcal{H}_{\infty}\right) \mid \mathcal{F}_{t}\right)=E\left(M_{\infty} \mid \mathcal{F}_{t}\right) .
\end{aligned}
$$

where $\chi_{A}$ is a indicator function for $A \in \mathcal{H}_{\infty}$, so

$$
M_{t}=E\left(M_{\infty} \mid \mathcal{F}_{t}\right)
$$

which means that elements of $H$ are $\left(\mathcal{F}_{t}\right)$ martingales.

Conversely, suppose that every $\left(\mathcal{H}_{t}, P\right)$-adapted $\left(\mathcal{G}_{t}, P\right)$-martingale is $\left(\mathcal{F}_{t}, P\right)$-martingale, i.e.

$$
\begin{gathered}
E\left(M_{\infty} \mid \mathcal{F}_{t}\right)=M_{t}=E\left(M_{\infty} \mid \mathcal{G}_{t}\right) \\
E\left(P\left(A \mid \mathcal{H}_{\infty}\right) \mid \mathcal{F}_{t}\right)=E\left(P\left(A \mid \mathcal{H}_{\infty}\right) \mid \mathcal{G}_{t}\right) \\
E\left(E\left(\chi_{A} \mid \mathcal{H}_{\infty}\right) \mid \mathcal{F}_{t}\right)=E\left(E\left(\chi_{A} \mid \mathcal{H}_{\infty}\right) \mid \mathcal{G}_{t}\right) \\
E\left(\chi_{A} \mid \mathcal{F}_{t}\right)=E\left(\chi_{A} \mid \mathcal{G}_{t}\right) \\
P\left(A \mid \mathcal{F}_{t}\right)=P\left(A \mid \mathcal{G}_{t}\right)
\end{gathered}
$$

for $A \in \mathcal{H}_{\infty}$ and also the definition of causality is satisfied, so $\mathrm{H} K \mathrm{G}$; F; $P$.
The similar result is shown in (Florens \& Fougere, 1996) but expressed in terms of noncausality.

Corollary 3.2 Let $\mathrm{H} \subseteq \mathrm{F}$. Then $\mathrm{H} K \mathrm{H} ; \mathrm{F} ; P$. if and only if every element of $H$ is $\left(\mathcal{F}_{t}, P\right)$ - martingale.

PROOF. It is the special case of the previous theorem (if $\mathrm{G}=\mathrm{H}$ ).

As the special case we can observe the process of Brownian motion (see Corollary 2.5) and Markovian process (see Lemma 2.4). By previous results we can conclude that the process which is its own cause is completely described by its behavior with respect to its natural filtration $\left(\mathrm{F}^{W}, \mathrm{~F}^{X}\right.$ respectively).

Also, the concept of causality can be connected and to the larger class of processes. Let us recall, the random process $\mathrm{N}=\left\{N_{t}, t \in I\right\}$ is called a local martingale with respect to the filtration $\left(\mathcal{F}_{t}\right)$ on the probability space $(\Omega, \mathcal{F}, P)$ if there exist an increasing sequence of stopping times $\left(T_{n}\right), n=1,2, \ldots$, such that $P\left(T_{n} \leq n\right)=1, P\left(\lim T_{n}=\infty\right)=1$ and for any $n=1,2, \ldots$, the sequences $\left\{N_{t \wedge T_{n}}\right\}$ are uniformly integrable martingales.

Let $G$ be a set of right continuous modifications of the $\left(\mathcal{G}_{t}\right)$-adapted processes of the form:

$$
G=\left\{N_{t}: N_{t}=P\left(A \mid \mathcal{G}_{t}\right), A \in \mathcal{G}_{\infty}\right\}
$$

Then, we have the following result.

Theorem 3.3 G $\subseteq$ F. Then G $K \quad$ G; F; $P$ if and only if every $\left(\mathcal{G}_{t}, P\right)$-local martingale is $\left(\mathcal{F}_{t}, P\right)$-local martingale.

ProOF. Suppose that G $K \quad$ G; F; $P$ holds, i.e.

$$
\left(\forall A \in \mathcal{G}_{\infty}\right) \quad P\left(A \mid \mathcal{G}_{t}\right)=P\left(A \mid \mathcal{F}_{t}\right) .
$$

Also, suppose that process $N_{t}$ is of the form (3), and that $N_{t}$ is a $\left(\mathcal{G}_{t}, P\right)$-local martingale. Then there exists a sequence $\left(T_{n}\right)$, of stopping times with respect to $\mathrm{G}$ for which $N_{t \wedge T_{n}}$ is a $\left(\mathcal{G}_{t}, P\right)$-martingale, for each n. According to Theorem 3 in (Bremaud \& Yor, 1978) the sequence $\left(T_{n}\right)$, of stopping times with respect to $\mathrm{G}$ is a sequence of stopping times with respect to $\mathrm{F}$ too. Due to Theorem 3.1, for each $n$ every $\left(\mathcal{G}_{t}, P\right)$ martingale $N_{t \wedge T_{n}}$, is $\left(\mathcal{F}_{t}, P\right)$ - martingale. Considering that $\left(T_{n}\right)$ are stopping times with respect to $\mathrm{F}$, the process $N_{t}$ is $\left(\mathcal{F}_{t}, P\right)$ - local martingale.

Conversely, suppose that every $\left(\mathcal{G}_{t}, P\right)$-local martingale is $\left(\mathcal{F}_{t}, P\right)$ - local martingale. If the assumption is true for every local martingale, then it is true for martingales (because every martingale is local martingale), and according to Theorem 3.1 we have $G$ K G; F; $P$. 


\section{ACKNOWLEDGMENT}

The work is supported by Serbian Ministry of Science and Technology.

\section{REFERENCES}

Bremaud, P., \& Yor, M. 1978. Changes of filtrations and of probability measures. Z. Wahrscheinlichkeitstheorie verw. Gebiete, 45(4), pp. 269-295, doi:10.1007/BF00537538.

Chamberlain, G. 1982. The General Equivalence of Granger and Sims Causality. Econometrica, 50(3), pp. 569-581, doi:10.2307/1912601.

Florens, J. P., \& Fougere, D. 1996. Noncausality in Continuous Time. Econometrica, 64(5), p. 1195 , doi: $10.2307 / 2171962$.

Florens, J. P., \& Mouchart, M. 1982. A Note on Noncausality. Econometrica, 50(3), pp. 583-591, doi:10.2307/1912602.

Florens, J. P., \& Mouchart, M. 1985. A Linear Theory for Noncausality. Econometrica, 53(1), pp. 157-175, doi: $10.2307 / 1911729$.

Granger, C. W. J. 1969. Investigating Causal Relations by Econometric Models and Crossspectral Methods.Econometrica, 37(3), pp. 424-438, doi: $10.2307 / 1912791$.

Granger, C. W. J., \& Newbold, P. 1977. Forecasting Economic Time Series.New York: Academic Press.

Gill, J. B., \& Petrović, Lj. 1987. Causality and Stochastic Dynamic Systems. SIAM J. Appl. Math., 47, pp. 1361-1366.

Hoover, D., \& Keisler, J. 1984. Adapted probability distributions. Trans. Amer. Math. Soc., 286, pp. 159201.

Hosoya, Y. 1977. On the Granger Condition for Noncausality. Ecomometrica, 45, pp. 735-1736.
Mykland, P. A. 1986. Statistical Causality. Statistical Report, University of Bergen, 4, pp. 1-28.

Mccrorie, J. R., \& Chambers, M. J. 2006. Granger causality and sampling of economic processes. Journal of Econometrics, 132, pp. 311336.

Petrović, Lj. 1989. Causality and Stochastic Realization Problem. Publ. Inst. Math., Beograd, 45(59), pp. 203-212.

Petrović, Lj. 1996. Causality and Markovian Representations. Statistics and Probability Letters, 29, pp. 223-227.

Petrović, Lj., \& Stanojević, D. 2005. Some Models of Causality and Weak Solutions of Stochastic Differential Equations with Driving Semimartingales. Facta Universitatis (Niš), Ser. Mathematics and Informatics, 5, pp. 103-112.

Petrović, Lj., \& Stanojević, D. 2010. Statistical Causality, Extremal Measures and Weak Solutions of Stochastical Differential Equations With Driving Semimartingales, J. Math. Model. Algor, 9, pp. 113128.

Petrović, Lj., \& Dimitrijević, S. 2011. Invariance of Statistical Causality Under Convergence. Statistic and Probability Letters, 81, pp. 1445-1448.

Petrović, Lj., \& Valjarević, D. 2013. Statistical Causality and Stable subspaces of Hp. Bulletin of Australian Mathematical Society, 88, pp. 17-25.

Rozanov, Y. A. 1974. Theory of Innovation Processes. In Monographs in Probability Theory and Mathematical Statistics.Moscow: Izdat, Nauka.

Rozanov, Y. A. 1977. Innovation Processes.New York: V. H. Winston and Sons..

Valjarević, D., \& Petrović, Lj. 2012. Statistical Causality and Orthogonality of Local Martingales. Statistic and Probability Letters, 82, pp. 1326-1330.

\footnotetext{
${ }^{*}$ E-mail: dragana.valjarevic@pr.ac.rs
} 